\title{
Fundus Camera Imaging and Mobile Phone Opthalmoscopy for Identification of Diabetic Retinopathy
}

Nikita kashyap, PhD Scholar, Dr. C.V.Raman University, Bilaspur, India, nikita2010k@gmail.com

Dr. Dharmendra Ku. Singh, Associate Professor (EEE), Dr. C.V.Raman University, Bilaspur, India, dmsingh2001@ rediffmail.com

Dr. Girish Ku. Singh, Assistant Professor (CSE), Dr. Harisingh Gour University, Sagar, India, gkrsingh@gmail.com

Abstract Diabetic Retinopathy (DR) is the retinal abnormalities arise on diabetic patients. Initial retinal screening is the super approach to prevent from diabetic retinopathy. Fundus imaging with good quality and large field is a popular method for DR identification and prevention. In this paper two fundus imaging methods are simulated. The first method is based on fundus camera which is used in the hospital to capture the retina image and In the second method Mobile phone with good camera quality, light-emitting diode (LED) and 28D lens and is used as an indirect ophthalmoscope. The results are shown and compared in terms of precision, recall and accuracy.

Keywords - Diabetic retinopathy, mobile phone, fundus camera, artificial neural network, discrete wavelet transform, retinal images.

\section{INTRODUCTION}

Diabetes is a long term organ disorder which is starts with increasing of blood sugar level and weak formation of hormone and allows sugar to enter in human body. Due to diabetes, amount of sugar in small blood vessels of eye cause difficult in vision and cause blindness known as DR (Diabetic Retinopathy) [1,2]. DR blocks and distorts the blood vessels of retina result in fluid liking and vision distorting. After 10 to 15 years of diabetes about $30 \%$ of patients develop serious visual impairment [3].

There are four stages of Diabetic retinopathy. With time the stages of disease increases from normal to 1st DR stage and 1 st to further advance stage. The stages for diabetic retinopathy are defined below [4-6]:

a). Mild non-proliferative DR: this in the first stage of diabetic retinopathy the eye swells which is known. known as aneurysm.

b). Moderate non-proliferative DR: In this stage the blood vessels started to distort, swelling increases and creates dotand-blot hemorrhage.

c). Severe non-proliferative DR: with the onset of disease the blocking of blood vessels increases are started and yellow spots called cotton wool spots are stated to create on retina side.

d). Proliferative diabetic retinopathy: The last stages of DR, new but fragile blood vessels are created on eye side.
Resulting in leakage of vessel and growing of this vessel will become the reason of serious visual impairment [7].

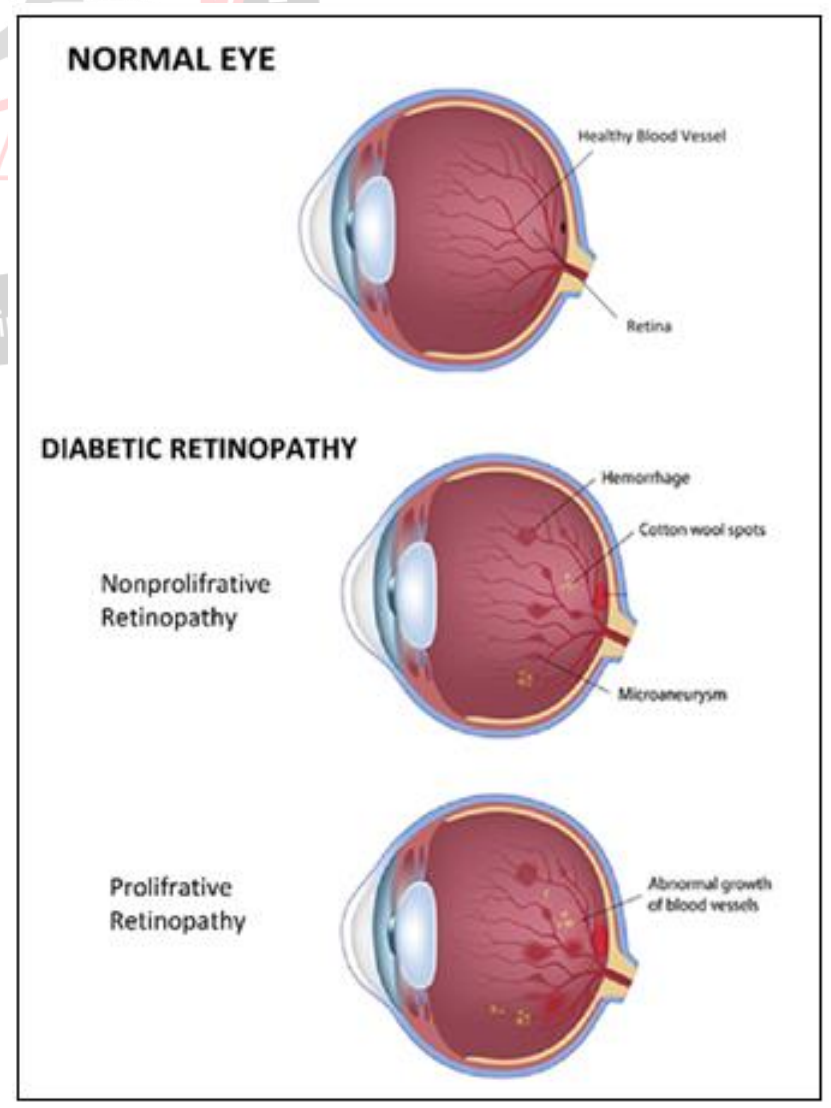

Fig. 1. Retinal image 
Early identification of DR reduces the chance of visual impairment [8]. The number of diabetic patients outnumber the number of eye specialist, so an early DR detection technique is needed so that only DR affected person go to the hospital for treatment and analysis $[9,10]$.

In this paper we used an automated detection system to identify the DR stages. For the detection process the artificial neural network is used that uses the identifier and wide number of training set for specific results and extraction process is done by DWT [11,12]. We also used the method in which the mobile phone and 28D condensing lens was used for retina imaging and compare this method with fundus camera imaging.

\section{METHOD OF RETINAL IMAGE ACQUISITION}

\section{A. Collecting retinal images by fundus camera}

A fundus camera is used in the hospitals to take the picture of the eye's inner part- retina, optic disc, macula and posterior pole which provide the detail information about the eye's health and monitor the eye abnormalities [13]. To capture picture firstly tell the patient to sit and place their forehead in the bar. Then the examiner open the cover of camera to illuminate the light on eye which lighting up the retina and take a picture of it [14].

Eye specialist operates these retinal cameras to identify, analyze treatment, and cure the eye related diseases [15]. Fundus images used in this research work is taken by using the Topcon mydriatic TRC-50DX fundus camera (as shown in fig. 2) with a $50^{\circ}, 35^{\circ}$ and $20^{\circ}$ field of view. It gives maximum resolution of $70 \mu \mathrm{m}$ and can amplify the image by 15 times. This type of camera gives upright, and more magnified view with the smaller field and most commonly used during a regular eye examination $[16,17]$. Example of retinal images of different DR stages captured by fundus camera is given in fig. 3 .

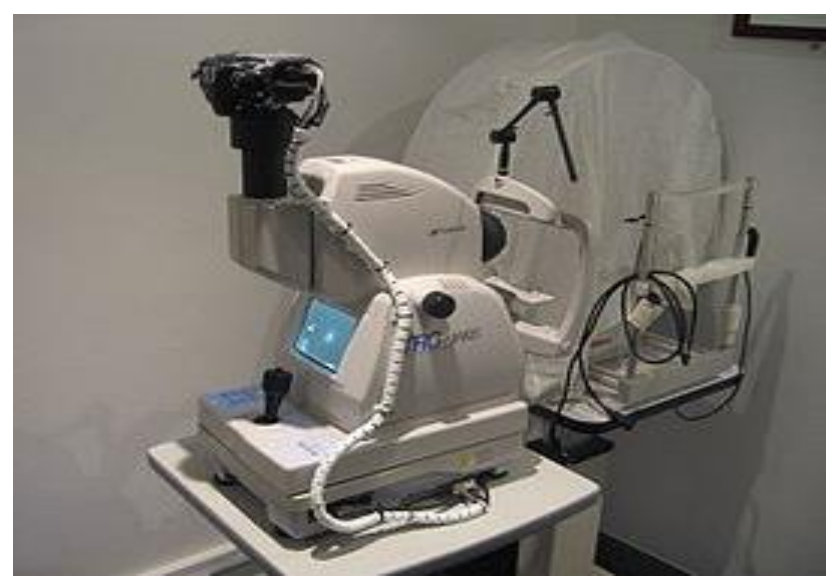

Fig.2: Topcon TRC-50DX fundus camera

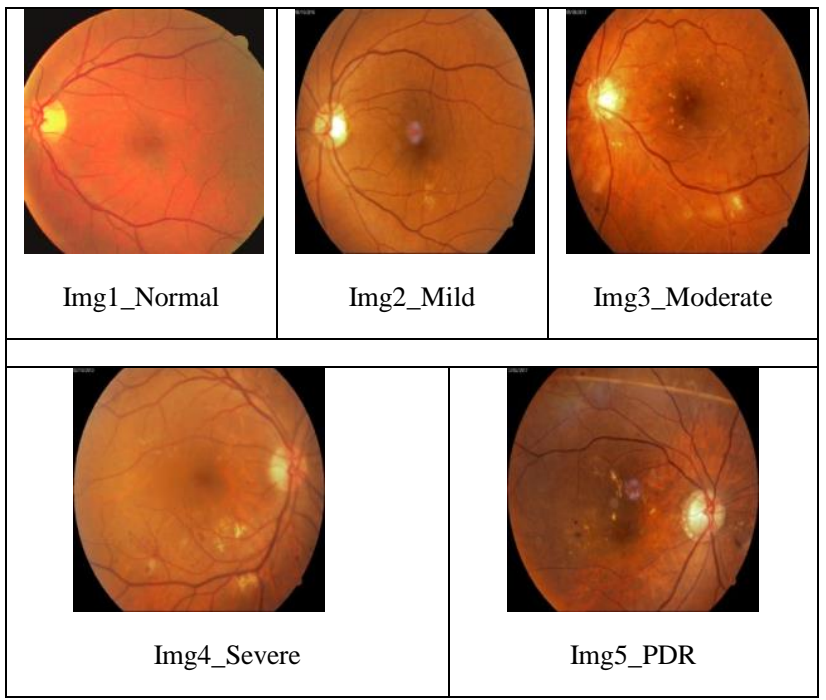

Fig.3. Retina image capture by fundus camera

\section{B. Collecting retinal images by Mobile phone}

For the detection and analysis of diabetic retinopathy the eye specialist use a fundus camera. However a fundus cameras are very costly, big and only skilled operators can operate it which are limited in number [18]. So there is need of a low cost handheld indirect ophthalmoscope which can be personally operated with little practice.

Now a days mobile phones have become an essential item of our life. It is very profitable, simple, handy, perform multiple operations and anybody can carry it at any place any time [19]. This causes great interest on mobile phone for medical application and examination like for ophthalmology as imaging devices. Mobile phones with good camera quality, light-emitting diode (LED) and 28D lens can be used in place of fundus camera to take the

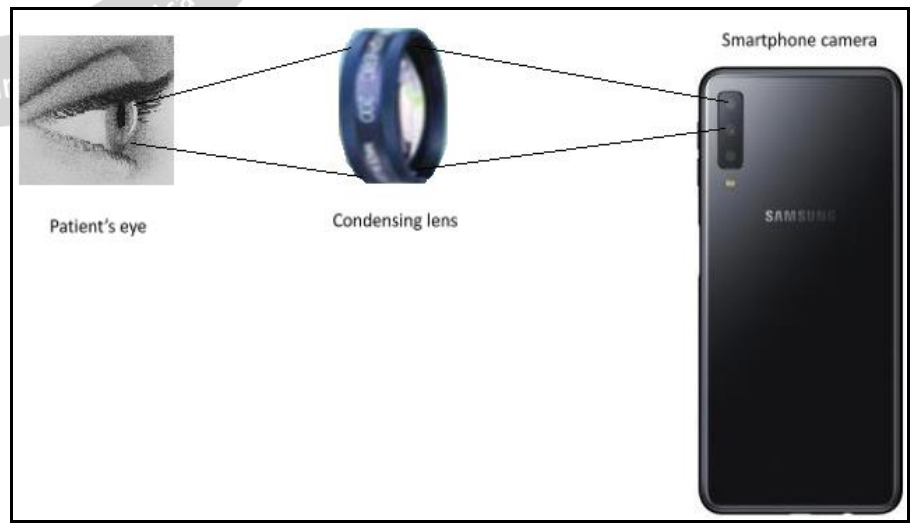

Fig.4. Principle of mobile phone indirect ophthalmology

We use Samsung Galaxy A7 2016 to capture the retina's picture. This phone has good camera quality (13MP) with LED flash, acceptable screen size, android operating system and cost is also affordable. To capture picture firstly tell the patient to lie flat on his back and stand near him then on one hand hold the lens 2 to 3 inch from patient's eye and in other hand hold mobile phone 20-22 inch from lens. Lighting up the retina by using Light Emitting Diode (LED) and take a picture of it [21]. Example of retinal images of 
different DR stages captured by mobile phone is given below:

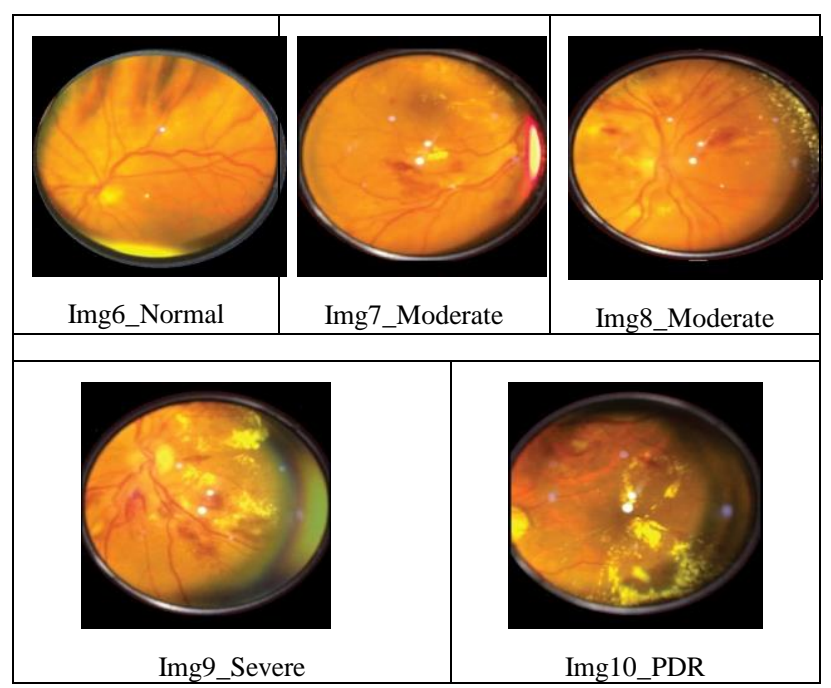

Fig 5. Retina images capture by mobile phone

\section{DR DETECTION USING ANN-DWT}

The ANN and DWT methods are used for DR detection. The database of diabetic retinopathy affected retinal image are created and patient's retina image capture by using a Fundus camera and Mobile phone. All the images are processed and converted into gray scale image.

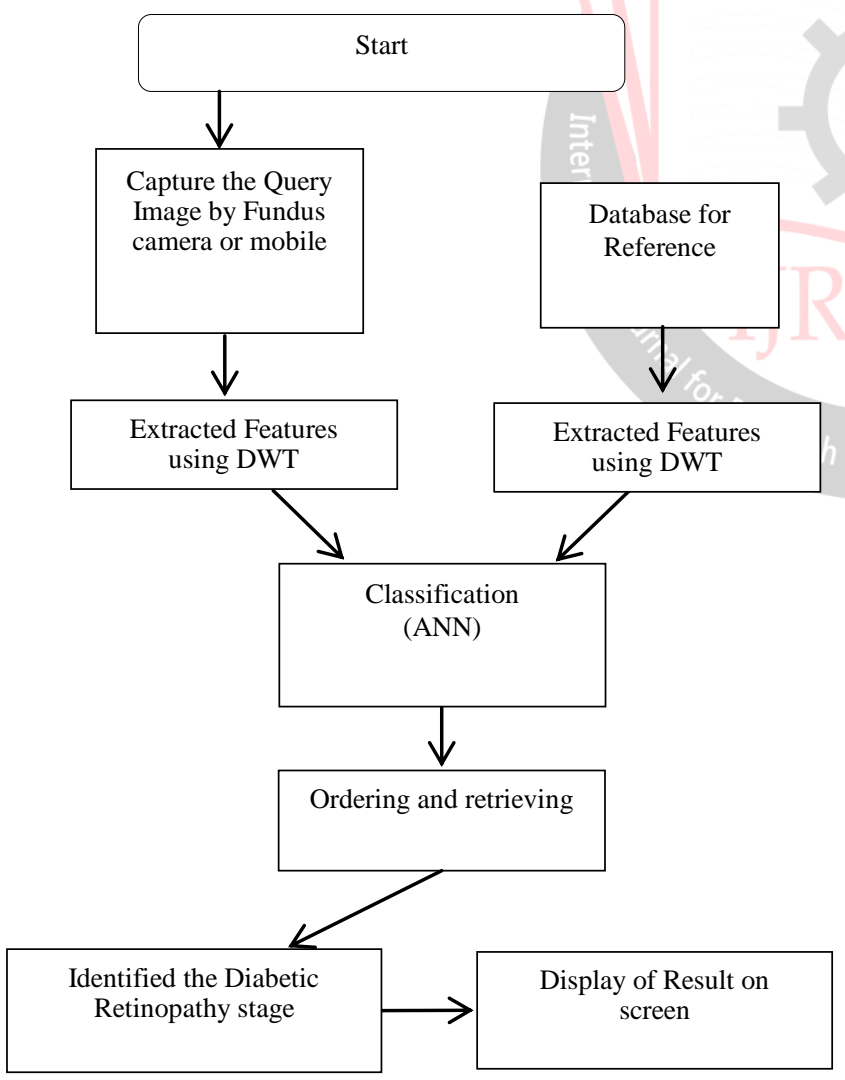

Fig 6. Flowchart of DR Detection Method Based on ANN-DWT

In the next step the DWT (Discrete Wavelet Transform) is applied which decompose the image into four sub-bands and extract the feature of images. At last apply this feature into ANN (Artificial Neural Network) model to find and classify the most relevant images to identify the DR stage.

To measure the performance of the system we calculate precision, recall and accuracy by following formulae [3]:

$$
\begin{gathered}
\text { Precision }=\frac{T P}{T P+F P} \\
\text { Recall }=\frac{T P}{T P+F N} \\
\text { Accuracy }=\frac{T P+T N}{T P+T N+F P+F N}
\end{gathered}
$$

Where, True Positive (TP): accurately identified DR images, True Negative (TN): accurately identified Non-DR images, False Positive (FP): Non-DR images identified wrongly as DR images, False Negative (FN): DR images identified wrongly as Non-DR images.

\section{EXPERIMENTAL RESULTS}

The Method is developed \& evaluated for a database CVRUDB1, CVRUDB2 and CVRUDB3 (as illustrated in table 1) which contains both normal and diabetic retinopathy affected eye image. These images are collected from three hospitals: Luthra Hospital Bilaspur, Shri Mahadevam Multispecialty Hospital Raipur, Aashirwad

\begin{tabular}{|c|c|c|c|}
\hline Database & CVRUDB1 & CVRUDB2 & CVRUDB3 \\
\hline $\begin{array}{l}\text { Total number of } \\
\text { Images }\end{array}$ & 112 & 56 & 466 \\
\hline Normal cases & 38 & 14 & 101 \\
\hline Mild NPDR & 31 & 19 & 134 \\
\hline Moderate NPDR & 28 & 15 & 110 \\
\hline Severe NPDR & 11 & 07 & 97 \\
\hline PDR & 04 & 01 & 24 \\
\hline Description & $\begin{array}{l}\text { Testing } \\
\text { Database, } \\
\text { Captured by } \\
\text { Fundus camera }\end{array}$ & $\begin{array}{l}\text { Testing } \\
\text { Database, } \\
\text { Captured by } \\
\text { Mobile Phone }\end{array}$ & $\begin{array}{l}\text { Training } \\
\text { Database, } \\
\text { Captured by } \\
\text { Fundus camera }\end{array}$ \\
\hline
\end{tabular}
Laser \& Phaco Eye Hospital Bilaspur.

Table 1: Description of Databases

The algorithm has been implemented on MATLAB R2016b software computing language. Table. 2 represents the result of classification while the table 2 shows the performance evaluation results (precision, recall and 
accuracy) of a database CVRUDB1 and CVRUDB2.

Table 2: Result of Classification

\begin{tabular}{|c|c|c|c|c|}
\hline Database & TP & TN & FP & FN \\
\hline CVRUDB1 & 68 & 36 & 2 & 8 \\
\hline CVRUDB2 & 35 & 12 & 2 & 7 \\
\hline
\end{tabular}

Table 3: Performance Evaluation Report

\begin{tabular}{|c|c|c|c|}
\hline Database & Precision & Recall & Accuracy \\
\hline CVRUDB1 & $97.14 \%$ & $89.47 \%$ & $92.86 \%$ \\
\hline CVRUDB2 & $94.59 \%$ & $83.33 \%$ & $83.92 \%$ \\
\hline
\end{tabular}

From these results it could be simply observed that the value of precision, recall and accuracy are less for database CVRUDB2 as compared to CVRUDB1. The lower performance is primarily due to the fact that the images of database CVRUDB2 were captured by mobile phone whose quality was compromised to some extent as the flash of the mobile phone camera is not a focused beam as in an fundus camera and there is a reflections from the condensing lens which reduce the clarity of the images. The comparison has been plotted below:

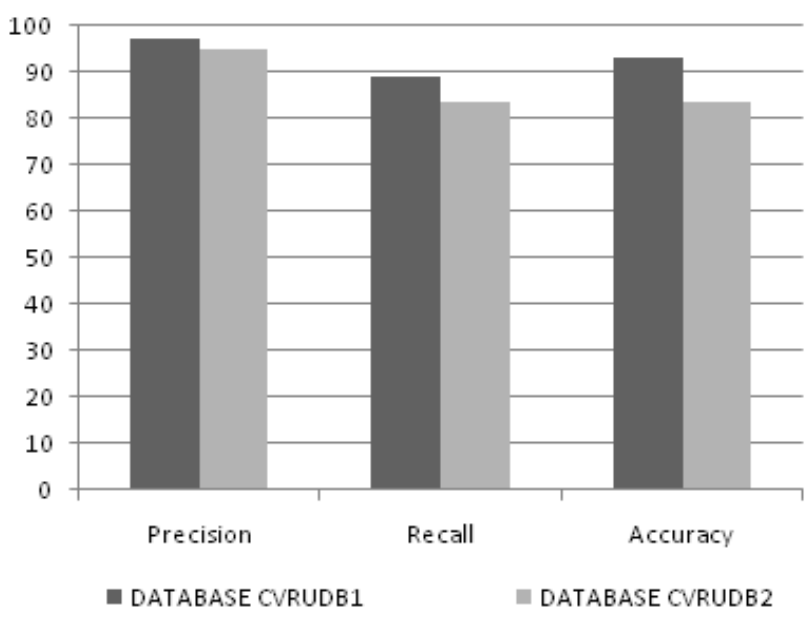

Fig 7: Performance Graph

\section{Conclusion}

We have used an automated DR identification model based on DWT \& ANN which is experimented with both fundus camera and mobile camera imaging. The experiment result shows that the fundus camera imaging provide high accuracy, precision, Recall rate and less analysis time for detecting the presence or absence of DR of any grade as compared to Mobile camera imaging. So the Mobile phone cameras are not capable to entirely replace the fundus cameras but it can be used in the area where the fundus cameras are not available. It reduce the cost of the detection system, doesn't need skilled operator and can be available at any time, any place ideal for rural regions.

\section{ACKNOWLEDGMENT}

I would like to acknowledge Dr. Priti Chandrawanshi (Shree mahadevam Multispecialty hospital Raipur), Dr. Luthra (Luthar hospital Bilaspur) and Dr. Madharia (Aashirwad Laser \& Phaco Eye Hospital Bilaspur) for providing me retina image of patients for the study.

\section{REFERENCES}

[1] DC Klonoff, DM Schwartz, "An economic analysis of interventions for diabetes", Diabetes Care, Vol. 23, pp.390-404, 2000.

[2] Zeljković Vesna, Milena Bojic, Shengwei Zhao, Claude Tameze and Ventzeslav Valev, "Exudates and optic disk detection in retinal images of diabetic patients", Concurrency and Computation: Practice and Experience, vol. 27(1), pp. 172-192, 2015.

[3] N. Kashyap, D. K. Singh. "Color Histogram Based Image Retrieval Technique for Diabetic Retinopathy Detection”. Int. Con. for Convergence in Technology (I2CT) Siddhant College of Engineering, Pune India, pp. 799-802, DOI: 10.1109/I2CT.2017.8226238, 2017.

[4] Sivakumar, Ravindran, Velmurughendran, Muthayya \& Lakshminarayanan "Diabetic Retinopathy Classification", IEEE Conference on Convergent Technologies for the AsiaPacific Region. Vol. 1, pp. $205-208,2003$.

[5] Rema M \& Pradeepa R., "Diabetic retinopathy: an Indian perspective". Indian journal of medical research, Vol. 125, no. 3, pp. 297, 2003.

[6] Kashyap N., Singh D.K. \& Singh G.K. "Review of Digital image processing techniques for detection of diabetic retinopathy", Int. Journal of Emerging Technologies and Innovative Research, Vol.6, Issue 3, page no. pp559-563, 2019.

[7] P. M. Rokade and R. R. Manza, "Automatic detection of hard exudates in retinal images using haar wavelet transform", International Journal of Application or Innovation in Engineering \& Management, 4, pp. 402410, 2015.

[8] Vallabha D., Dorairaj R, Namuduri K. R. and Thompson H., "Automated Detection and Classification of Vascular Abnormalities in Diabetic Retinopathy", 38th Asilomar Conference on Signals, Systems and Computers, Vol. 2, pp. 1625 - 1629, 2004.

[9] Jagadish N., P S Bhat, Rajendra Acharya U, Lim Choo Min, M. Gupta, “Automated Identification of Different 
Stages of Diabetic Retinopathy using digital fundus images", Journal of Medical Systems, USA, 2007.

[10] Kashyap N. and Sinha G. R., "Image Watermarking Using 2-Level DWT", Advances in Computational Research, Vol. 4, No.1, pp. 42-45, 2012.

[11] Kashyap N. and Sinha G. R., Image Watermarking Using 3-Level DWT", I. J. Modern Education and Computer Science, Vol. 3, pp. 50-56, 2012.

[12] Pavle Prentasic "Detection of Diabetic Retinopathy in Fundus Photographs", International computer engineering Conference, pp. 20 - 25, 2012.

[13] Parag S. Chandakkar, Ragav Venkatesan , Baoxin Li , Helen K. Li, "Retrieving clinically relevant diabetic retinopathy images using a multi-class multipleinstance framework", Proc. SPIE 8670, Medical Imaging Computer-Aided Diagnosis, 86700Q, doi:10.1117/12.2008133, 2013.

[14] Mohammad A. Al-Jarrah, Hadeel Shatnawi, "Nonproliferative diabetic retinopathy symptoms detection and classification using neural network". Journal of Medical Engineering \& Technology, 41 (6); pp. 498505; DOI: 10.1080/03091902.2017.1358772, 2017.

[15] Morgan Micheletti, J \& Hendrick, Andrew \& N Khan, Farah \& C Ziemer, David \& Pasquel, Francisco, "Current and Next Generation Portable Screening Devices for Diabetic Retinopathy". Journal of diabetes science and technology, DOI: 10. 10.1177/1932296816629158, 2016.

[16] Sandeep Bhat; Malavika Bhaskaranand; Chaithanya Ramachandra; Todd P Margolis; Daniel A. Fletcher; Kaushal Solanki, "Fully-automated Diabetic Retinopathy Screening Using Cellphone-based Cameras", Investigative Ophthalmology \& Visual Science, Vol.56, 1428, 2015.

[17] Toy BC, Myung DJ, He L, Pan CK, Chang RT, Polkinhorne A, Merrell D, Foster D, Blumenkranz MS., "Smartphone-Based Dilated Fundus Photography and Near Visual Acuity Testing as Inexpensive Screening Tools to Detect Referral Warranted Diabetic Eye Disease". Retina the journal of retinal and vitreous diseases, Vol. 0, No. 0, pp. 1-9, 2016.

[18] Nikita Kashyap, Dr. Dharmendra Kumar Singh and Dr. Girish Kumar Singh, "Develop a Novel Screening Tool Based on Wavelet Transform for Identification of Diabetic Retinopathy". Anusandhan (AUJ-AN), Vol 9, issue 17, pp.41-46, 2019.

[19] Nikita Kashyap, Dharmendra Kumar Singh and Girish Kumar Singh. Mobile Phone Based Diabetic Retinopathy Detection System Using ANN-DWT. 4th IEEE UPCON Conference on Electrical, Computer and Electronics, GLA University Mathura India. pp. 463-467, DOI:10.1109/UPCON.2017.8251092, 2019.

[20] Nikita Kashyap, Dharmendra Kumar Singh and Girish Kumar Singh, "Color Histogram- and SmartphoneBased Diabetic Retinopathy Detection System", ICoEVCI, Vol. 478, 669-678. 10.1007/978-981-131642-5_58, 2018.

[21] Nikita Kashyap, Dharmendra Kumar Singh and Girish Kumar Singh, "A Novel Approach for Detecting and Grading Diabetic Retinopathy using Wavelet Transform and Artificial Neural Network". IJRECE. Vol. 7, Issue 2, pp. 100-104, 2019. 